\title{
Synchronous ovarian metastasis from colorectal cancer: A report of two cases
}

\author{
JIRO SHIMAZAKI, TAKANOBU TABUCHI, \\ KIYOTAKA NISHIDA, AKIRA TAKEMURA, GYO MOTOHASHI, HIDEKI KAJIYAMA and SHUJI SUZUKI \\ Department of Gastroenterological Surgery, Ibaraki Medical Center, Tokyo Medical University, \\ Ami, Ibaraki 300-0395, Japan
}

Received March 26, 2015; Accepted April 18, 2016

DOI: $10.3892 / \mathrm{ol} .2016 .4553$

\begin{abstract}
Ovarian metastasis of colorectal cancer is relatively rare. The present study reports two cases of synchronous ovarian metastasis from colorectal cancer, which were managed by cytoreductive surgery. In case one, a 60-year-old female patient presented with a multilocular pelvic tumor and ascites. Virtual colonoscopy revealed a mass in the sigmoid colon; however, no tumor cells were identified on histological examination. Ovarian metastasis from sigmoid colon cancer was suspected and adnexectomy was subsequently performed. Histological examination of the excised tumor revealed adenocarcinoma. Immunohistochemical analysis of the resected tumor revealed positive staining for cytokeratin (CK)20 and caudal-type homeobox 2 (CDX2), and negative staining for $\mathrm{CK} 7$, estrogen receptor, progesterone receptor and inhibin. The immunohistological results supported the diagnosis of ovarian metastasis from sigmoid colon cancer. In case two, a 56-year-old female patient presented with a multilocular pelvic tumor and ascites. Colonoscopy identified a rectal tumor, and histological examination revealed moderately-differentiated adenocarcinoma, which was confirmed by cytological analysis of ascites. Subsequently, ovarian metastasis from rectal cancer with peritoneal dissemination was diagnosed, and left ovariectomy and transverse colostomy were performed. Histological examination of the excised tumor revealed moderately-differentiated adenocarcinoma, and immunohistochemical investigation revealed positive staining for $\mathrm{CK} 20$ and CDX2, but negative staining for CK7. These immunohistological results indicated ovarian metastasis from rectal cancer. Both patients recovered well and are currently undergoing regular follow-up examinations. The observations from the two cases indicate
\end{abstract}

Correspondence to: Dr Jiro Shimazaki, Department of Gastroenterological Surgery, Ibaraki Medical Center, Tokyo Medical University, 3-20-1 Chuo, Ami, Inashiki, Ibaraki 300-0395, Japan E-mail: shima-j@tokyo-med.ac.jp

Key words: colorectal cancer, ovarian metastasis, pelvic tumor, surgery, prognosis that ovarian metastases of primary colorectal cancer may present as pelvic tumors and, thus, preoperative examination of the gastrointestinal tract is required. Furthermore, even in cases of widespread colorectal cancer metastases, excision of the ovarian tumor is required to establish a histological diagnosis for the selection of appropriate treatments.

\section{Introduction}

Common sites for synchronous metastases from colorectal cancer include the liver, lung, peritoneum, bone and brain (1). The frequency of ovarian metastasis from colorectal cancer is $1.6-6.4 \%$, however, this type of metastasis is difficult to distinguish from primary ovarian neoplasms (2-5). Furthermore, synchronous ovarian metastasis from colorectal cancer is generally poor, and the optimal first-line treatment strategy is debatable $(6,7)$. The present study reports two cases of synchronous ovarian metastasis from colorectal cancer that were managed by cytoreductive surgery.

\section{Case report}

Case one. A 60-year-old female patient presented to Katsuta Hospital (Katsuta, Japan) in June 2014 with progressive abdominal distension and lower abdominal pain. The following day the patient was referred to Ibaraki Medical Center, Tokyo Medical University (Ami, Japan) with a suspected diagnosis of pelvic tumor. The patient's medical history was otherwise unremarkable. Physical examination revealed lower abdominal tenderness with a palpable mass. Laboratory data revealed slight hypoalbuminemia (albumin, $3.5 \mathrm{~g} / \mathrm{dl}$; normal range, $>4.0 \mathrm{~g} / \mathrm{dl}$ ), and carcinoembryonic antigen (CEA; $11.1 \mathrm{ng} / \mathrm{ml}$; normal range, $<5.0 \mathrm{ng} / \mathrm{ml}$ ) and carbohydrate antigen (CA) 125 (743.7 U/ml; normal range, $<37.0 \mathrm{U} / \mathrm{ml}$ ) levels were increased. Abdominal computed tomography (CT; Somatom Sensation Cardiac; Siemens, AG, Munich, Germany) revealed a multilocular cystic pelvic mass with a solid component measuring $17 \mathrm{~cm}$ in diameter and an irregular mass located in the sigmoid colon (Fig. 1). Extensive ascites were also present. Virtual colonoscopy (Synapse VINCENT; Fujifilm Corporatio, Tokyo, Japan) revealed stenosis with a mass in the sigmoid colon (Fig. 2), which was confirmed by traditional colonoscopy. However, no tumor cells were identified in the biopsy specimen 
(which was obtained during colonoscopy) on hematoxylin and eosin histological examination. Ovarian metastasis from sigmoid colon cancer was suspected, therefore, adnexectomy was performed in July 2014. Intraoperatively, a disseminated tumor in the pelvic cavity was identified and cytology of the ascites using Papanicolaou staining revealed clusters of atypical cells exhibiting anisokaryosis, hyperchromasia and enlarged nuclei, yielding a diagnosis of adenocarcinoma. The resected tumor measured $17 \times 14 \times 8 \mathrm{~cm}$, and macroscopic examination revealed multicystic walls and septa composed of solid and necrotic components (Fig. 3A). Hematoxylin and eosin histological examination of the formalin-fixed and paraffin-embedded excised tumor revealed moderately-differentiated adenocarcinoma forming in the septa with infiltration of the cystic wall (Fig. 3B). Immunohistochemical analysis of the tumor revealed positive staining for cytokeratin (CK)20 (mouse monoclonal; dilution 1:50; M7019; Dako, Glostrup Denmark) and caudal-type homeobox 2 (CDX2; rabbit monoclonal; dilution 1:1; 418011; Nichirei Biosciences, Inc., Tokyo, Japan), and negative staining for CK7 (mouse monoclonal; dilution 1:100; M7018; Dako), estrogen receptor (rabbit monoclonal; dilution 1:1; 107925; Roche Diagnostics, Basel, Switzerland), progesterone receptor (rabbit monoclonal; dilution 1:1; 109431; Roche Diagnostics) and inhibin (mouse monoclonal; dilution 1:50; M3609; Dako) (Fig. 4). These immunohistological results supported the diagnosis of ovarian metastasis originating from colon cancer of the sigmoid. Recovery was uneventful and the patient was discharged 12 days after surgery. From October 2014, the patient was administered modified FOLFOX6 [oxaliplatin $\left(85 \mathrm{mg} / \mathrm{m}^{2}\right)$, leucovorin $\left(400 \mathrm{mg} / \mathrm{m}^{2}\right)$ and fluorouracil $(5-\mathrm{FU} ; 400 \mathrm{mg} /$ $\mathrm{m}^{2}$ ) intravenous infusion on day 1 , followed by $2,400 \mathrm{mg} / \mathrm{m}^{2}$ intravenous infusion of 5-FU over $46 \mathrm{~h}$ every 2 weeks] plus anti-vascular endothelial growth factor monoclonal antibody (bevacizumab; $5 \mathrm{mg} / \mathrm{kg}$ every 2 weeks) for primary sigmoid colon cancer with peritoneal dissemination. At present, the patient is regularly followed up every 2 weeks at the outpatient clinic of Ibaraki Medical Center, Tokyo Medical University, and her condition remains stable at the time of writing the present manuscript, in April 2016.

Case two. A 56-year-old female patient presented to Ryugasaki Saiseikai Hospital (Ryugasaki, Japan) in September 2014 with progressive abdominal distension. The following day the patient was referred to Ibaraki Medical Center, Tokyo Medical University, with a suspected ovarian tumor. The patient's medical history was otherwise unremarkable. Physical examination revealed abdominal distention with fluctuation, indicating abundant ascites. Laboratory data revealed increased lactate dehydrogenase $(2,473 \mathrm{IU} / \mathrm{l}$, normal range, 120-240 IU/l), CEA (93.9 ng/ml) and CA 125 (274.4 U/ml) levels. Abdominal CT (Somatom Sensation Cardiac) revealed a multilocular cystic pelvic mass with a solid component, measuring $12 \mathrm{~cm}$ in diameter, and ascites (Fig. 5). Cytology of abdominocentesis fluid using Papanicolau staining revealed clusters of atypical cells exhibiting anisokaryosis, hyperchromasia and enlarged nuclei, thus confirming adenocarcinoma, while colonoscopy revealed an elevated tumor with a central depression in the rectum, which did not involve the tumor. Biopsy of the tumor specimen
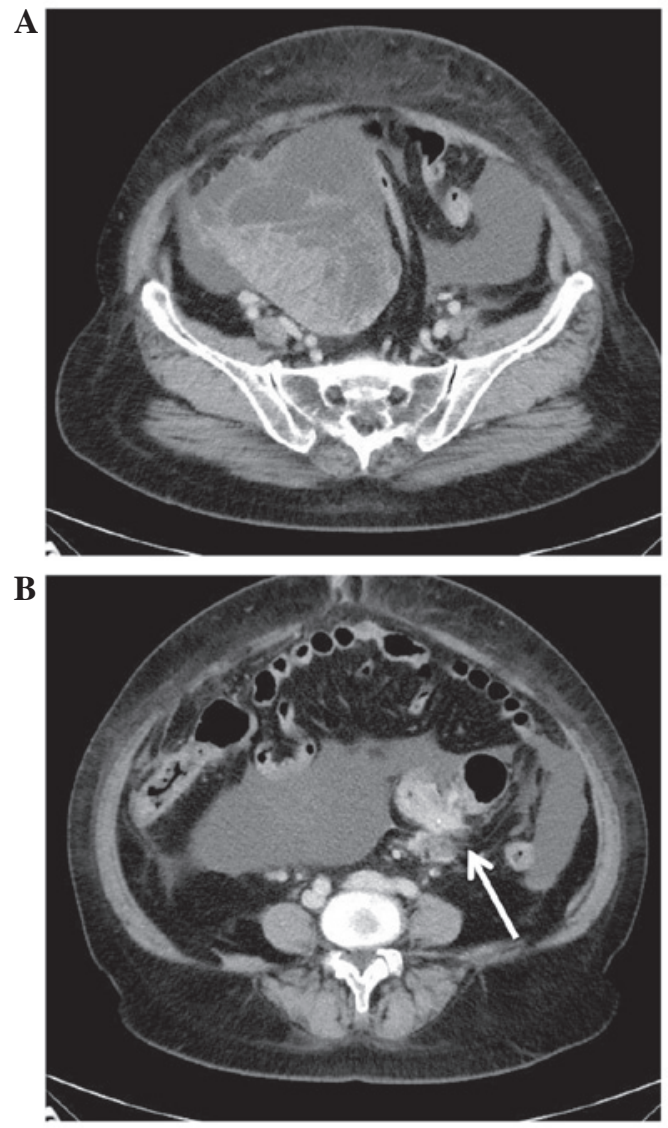

Figure 1. Case one. Abdominal enhanced computed tomography demonstrating (A) a multilocular cystic mass in the pelvic space and (B) an irregular mass located in the sigmoid colon (arrow).

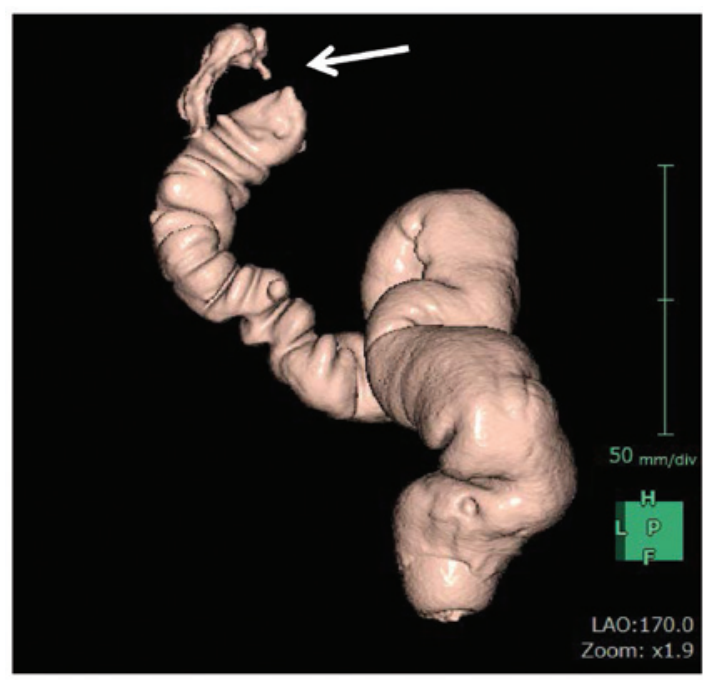

Figure 2. Case one. Virtual colonoscopy revealing stenosis with a mass in the sigmoid colon (arrow).

indicated moderately-differentiated adenocarcinoma. A diagnosis of ovarian metastasis from rectal carcinoma with peritoneal dissemination was established; therefore, left ovariectomy and transverse colostomy were performed in November 2014. The resected tumor measured $12 \times 11 \times 8 \mathrm{~cm}$, and macroscopic examination revealed multicystic walls and septa composed of a solid component with hemorrhage 

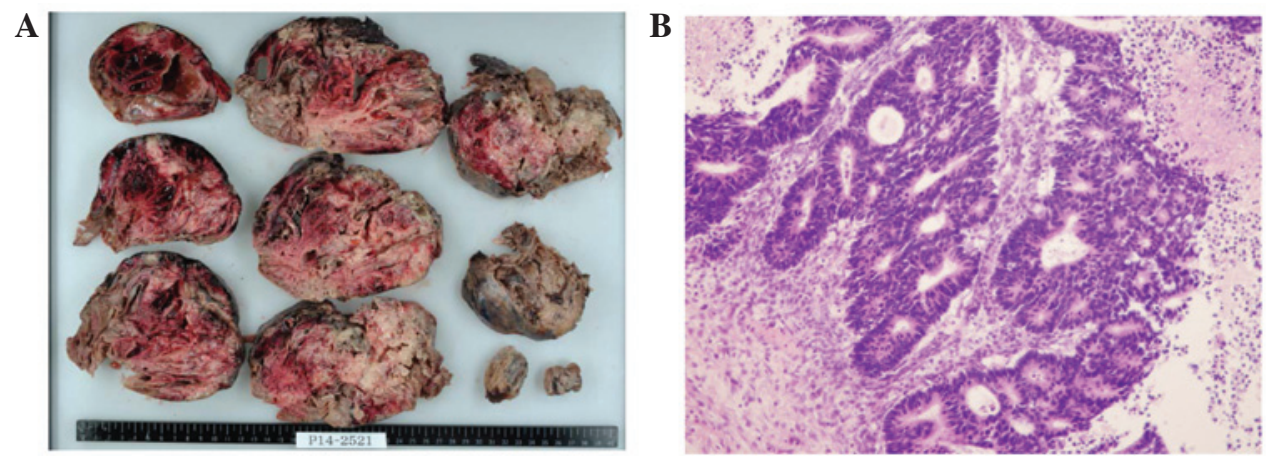

Figure 3. Case one. Macroscopic examination and histological analysis of the excised tumor specimen. (A) A multilocular cystic tumor, measuring $17 \mathrm{x} 14 \mathrm{x} 8 \mathrm{~cm}$ with multicystic walls and septa, containing solid and necrotic components was identified. (B) Histological staining revealing moderately-differentiated adenocarcinoma forming in the septa with infiltration of the cystic wall (stain, hematoxylin and eosin; magnification, $\mathrm{x} 100$ ).
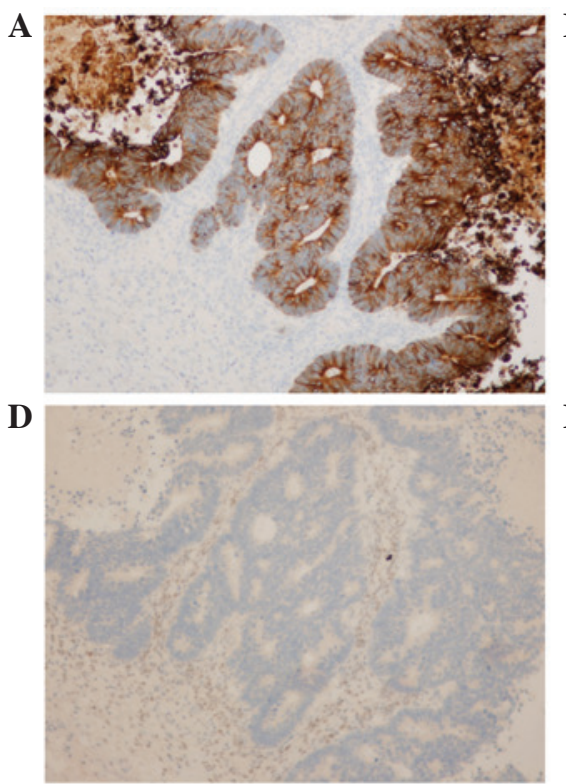

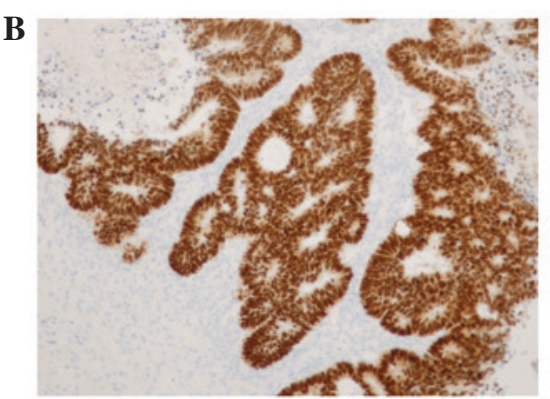

$\mathbf{E}$

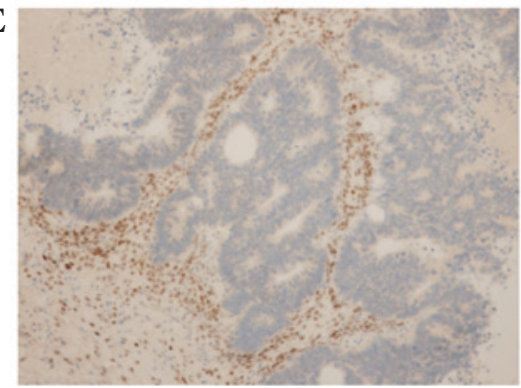

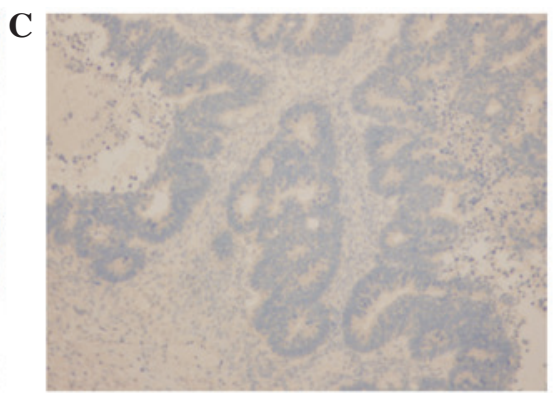

$\mathbf{F}$

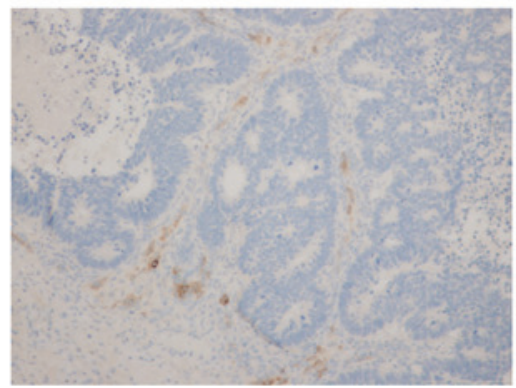

Figure 4. Case one. Immunohistochemical staining of the excised tumor revealed positive staining for (A) cytokeratin (CK)20 and (B) caudal-type homeobox 2 , and negative staining for (C) CK7, (D) estrogen receptor, (E) progesterone receptor and (F) inhibin (magnification, x100).

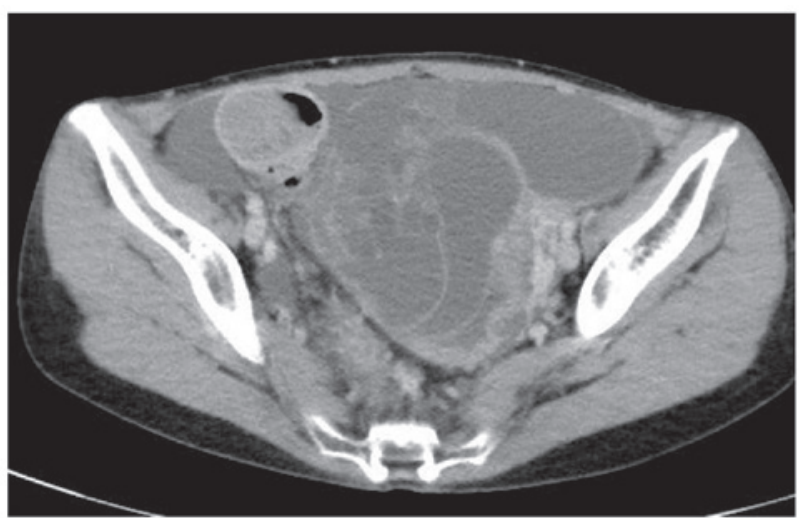

Figure 5. Case two. Abdominal enhanced computed tomography demonstrating a multilocular cystic mass and ascites in the pelvic space.

(Fig. 6). Hematoxylin and eosin histological examination of the formalin-fixed and paraffin-embedded excised tumor revealed moderately-differentiated adenocarcinoma (Fig. 7A), and

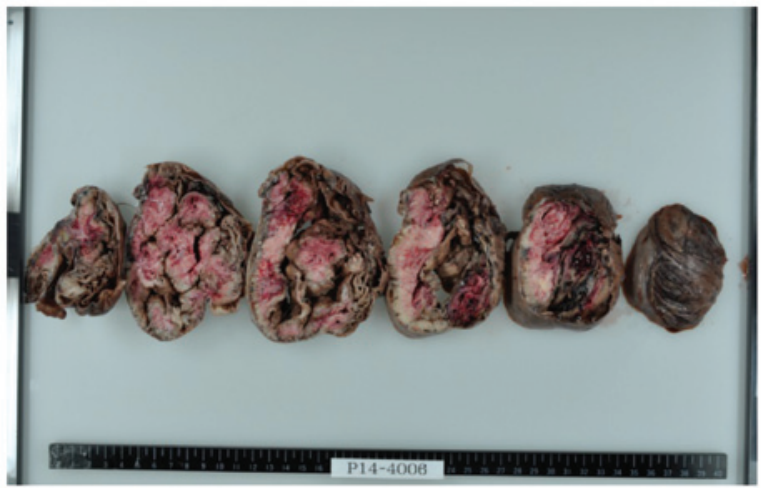

Figure 6. Case two. Macroscopic examination of the excised specimen revealing a multilocular cystic tumor measuring $12 \times 11 \times 8 \mathrm{~cm}$, with multicystic walls and septa, composed of solid and necrotic components.

immunohistochemical analysis identified positive staining for CK20 and CDX2, and negative staining for CK7 (Fig. 7B-D). These immunohistological results confirmed the diagnosis 


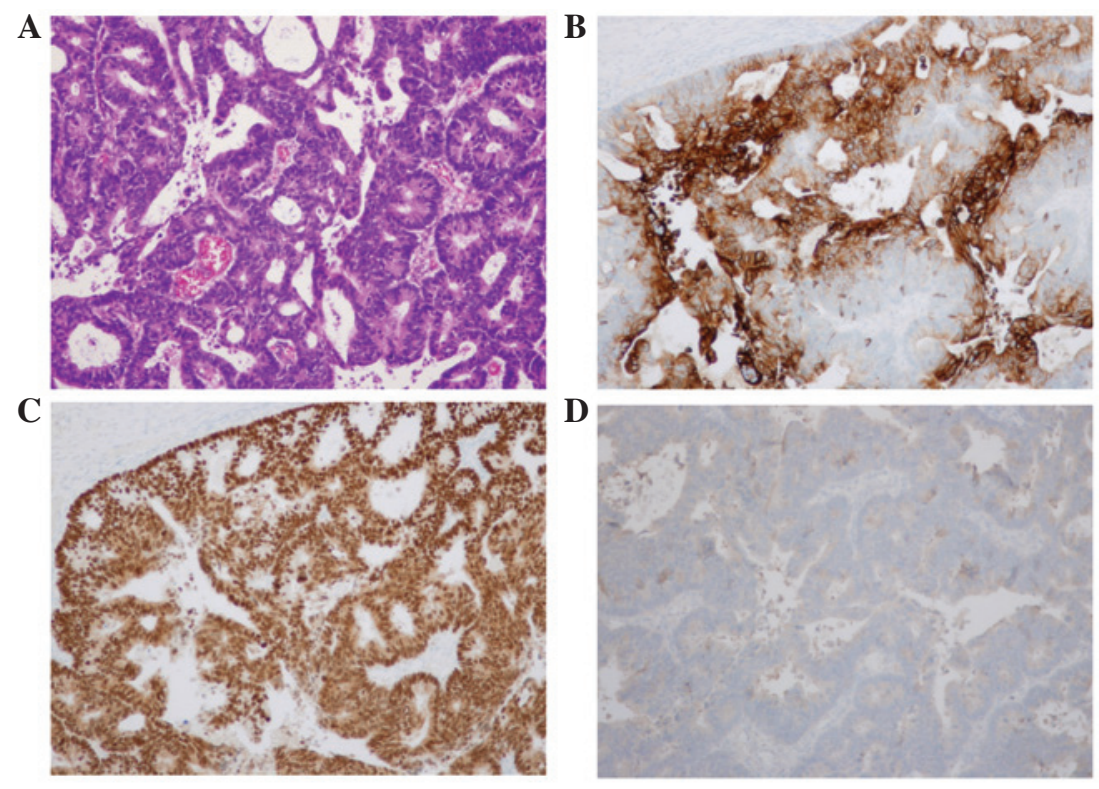

Figure 7. Case two. (A) Histological analysis of the excised tumor revealing moderately-differentiated adenocarcinoma forming in the cystic walls and septa (stain, hematoxylin and eosin; magnification, x100). Immunohistochemical staining of the excised tumor revealing positive staining for (B) cytokeratin 20 and (C) caudal-type homeobox 2 and (D) negative staining for CK7.

of ovarian metastasis from rectal cancer. Recovery was uneventful and the patient was discharged 13 days postoperatively. From December 2014, the patient was administered modified FOLFOX plus bevacizumab for primary rectal cancer with peritoneal dissemination. At present, the patient is undergoing regular follow-up examinations every 2 weeks at the outpatient clinic of Ibaraki Medical Center, Tokyo Medical University, and her condition was stable at the time of writing.

\section{Discussion}

Metastatic ovarian tumors account for $\sim 21.5 \%$ of all malignant ovarian tumors and 3.7-7.4\% of the cases metastasize from colorectal cancer (8-10). However, the process by which colorectal cancer metastasizes to the ovary remains unclear. Graffner et al (9) and Birnkrant et al (11) have postulated that, as there is no lymph flow between the colon and the ovaries, both hematogenous and disseminated peritoneal metastasis present possible metastatic pathways. In the two present cases, peritoneal dissemination was confirmed intraoperatively. Clinically, it is difficult to distinguish between primary and metastatic cancer of the ovary, which results in diagnostic problems for clinicians, radiologists and pathologists. Regarding radiological examination, Cho and Gold (12) reported that a mixed cystic and solid ovarian mass observed by $\mathrm{CT}$ scan must be regarded as a metastatic tumor in patients with a history of colonic or gastric carcinoma. In the present cases, the ovarian tumor presented as a multilocular cystic pelvic mass with a solid component. In addition, the patient in case two was preoperatively diagnosed with advanced rectal carcinoma with peritoneal dissemination. Regarding histological examination, Lee and Young (2) reported that bilaterality, microscopic surface involvement of epithelial cells and an infiltrative pattern of stromal invasion were strong indicators of metastatic ovarian carcinoma.
In case one, histological examination of the sigmoid colon tumor did not reveal carcinoma cells, although virtual colonoscopy identified stenosis with a mass. However, resection of the ovarian tumor revealed moderately-differentiated adenocarcinoma, and immunohistochemical analysis of the tumor cells revealed positive staining for CK20 and CDX2, and negative staining for CK7, estrogen receptor, progesterone receptor and inhibin. In the majority of cases, primary ovarian neoplasms exhibit positive staining for CK7 and negative staining for CK20. By contrast, colorectal carcinomas most frequently exhibit negative staining for CK7 and positive staining for CK20 $(13,14)$. CDX2 is a homeobox gene encoding the CDX2 protein, which serves as a transcription factor that is expressed in the nuclei of intestinal epithelial cells (15). CDX2 is a useful marker for adenocarcinoma of the gastrointestinal tract, and for distinguishing between primary and metastatic carcinomas of the ovary (16-18). According to immunohistological examination, the results of case one support the diagnosis of metastatic ovarian carcinoma from sigmoid colon carcinoma.

The optimal first-line treatment strategy for synchronous ovarian metastasis from colorectal cancer remains controversial. The Japanese guidelines for colorectal cancer treatment recommend surgery for metastatic lesions if the primary colorectal and metastatic lesions are completely resectable, and if the patient is able to tolerate resection of the metastatic lesions (1). In the present two cases, tumor dissemination was intraoperatively detected in the pelvic cavity, however, complete resection was not possible for all lesions. Only excision of ovarian metastases was performed for the following reasons: i) Patients presented with progressive abdominal distension and excision of ovarian metastasis may have alleviated the symptoms (19); ii) it is difficult to distinguish between primary and metastatic cancer of the ovary by diagnostic imaging alone, thus, a definitive histological diagnosis was required to identify appropriate treatment, particularly in 
case one (preoperative histological examination of the sigmoid colon tumor did not lead to a diagnosis); and iii) cytoreductive surgery is associated with improvement of overall survival in patients with widespread metastases of colorectal cancer (20). A number of cases of synchronous ovarian metastasis from colorectal cancer also exhibit distant metastasis and/or peritoneal dissemination $(6,7)$, therefore, the prognosis is generally poor. Jiang et al (21) reported that the median survival in patients with residual disease after metastasectomy is 10 months. However, as a result of marked improvement in systemic chemotherapy treatment for advanced colorectal cancer, it has been estimated that the median survival time of the patients may improve to $>20$ months following the administration of FOLFOX or 5-FU, leucovorin and irinotecan combination chemotherapy plus bevacizumab or anti-epidermal growth factor receptor monoclonal antibody (22-25).

In conclusion, ovarian metastases from primary colorectal cancer may present as pelvic tumors, thus, preoperative examination of the gastrointestinal tract and excision of the ovarian tumor are required to establish a histological diagnosis for the selection of appropriate treatment strategies.

\section{Acknowledgements}

The authors would like to thank Enago (www.enago.jp) for reviewing the English language of the present study.

\section{References}

1. Watanabe T, Itabashi M, Shimada Y, Tanaka S, Ito Y, Ajioka Y, Hamaguchi T, Hyodo I, Igarashi M, Ishida $\mathrm{H}$, et al; Japanese Society for Cancer of the Colon and Rectum: Japanese Society for Cancer of the Colon and Rectum (JSCCR) guidelines 2010 for the treatment of colorectal cancer. Int J Clin Oncol 17: 1-29, 2012

2. Lee KR and Young RH: The distinction between primary and metastatic mucinous carcinomas of the ovary: Gross and histologic findings in 50 cases. Am J Surg Pathol 27: 281-292, 2003.

3. Shiono S, Saito T, Fujii H, Arakawa A, Nakamura T and Yao T: A case of Krukenberg carcinoma metastasized from colon cancer resembling mucinous cystadenocarcinoma of the ovary. Int $\mathrm{J}$ Clin Exp Pathol 7: 394-401, 2013.

4. Aiyer R, Sweetman K, Larsen-Disney P and Fish A: A colorectal carcinoma imitating a primary ovarian carcinoma in a postpartum woman. BMJ Case Rep 2013: pii: bcr2013201055, 2013.

5. Hata T, Yoshioka S, Asukai K, Mizumoto S, Nakanishi M, Hamano R, Maekawa T, Hama N, Kashiwazaki M, Taniguchi M, et al: Two cases of ovarian metastasis of colon cancer. Gan To Kagaku Ryoho 38: 2286-2288, 2011 (In Japanese).

6. Chung TS, Chang HJ, Jung KH, Park SY, Lim SB, Choi HS and Jeong SY: Role of surgery in the treatment of ovarian metastases from colorectal cancer. J Surg Oncol 100: 570-574, 2009.

7. Rayson D, Bouttell E, Whiston F and Stitt L: Outcome after ovarian/adnexal metastectomy in metastatic colorectal carcinoma. J Surg Oncol 75: 186-192, 2000.
8. Ayhan A, Tuncer ZS and Bükülmez O: Malignant tumors metastatic to the ovaries. J Surg Oncol 60: 268-276, 1995.

9. Graffner HO, Alm PO and Oscarson JE: Prophylactic oophorectomy in colorectal carcinoma. Am J Surg 146: 233-235, 1983.

10. MacKeigan JM and Ferguson JA: Prophylactic oophorectomy and colorectal cancer in premenopausal patients. Dis Colon Rectum 22: 401-405, 1979.

11. Birnkrant A, Sampson J and Sugarbaker PH: Ovarian metastasis from colorectal cancer. Dis Colon Rectum 29: 767-771, 1986.

12. Cho KC and Gold BM: Computed tomography of Krukenberg tumors. AJR Am J Roentgenol 145: 285-288, 1985.

13. Wauters CC, Smedts F, Gerrits LG, Bosman FT and Ramaekers FC: Keratins 7 and 20 as diagnostic markers of carcinomas metastatic to the ovary. Hum Pathol 26: 852-855, 1995.

14. Berezowski K, Stastny JF and Kornstein MJ: Cytokeratins 7 and 20 and carcinoembryonic antigen in ovarian and colonic carcinoma. Mod Pathol 9: 426-429, 1996.

15. German MS, Wang J, Fernald AA, Espinosa R III, Le Beau MM and Bell GI: Localization of the genes encoding two transcription factors, LMX1 and CDX3, regulating insulin gene expression to human chromosomes 1 and 13. Genomics 24: 403-404, 1994.

16. Mutoh H, Sakurai S, Satoh K, Tamada K, Kita H, Osawa H, Tomiyama T, Sato Y, Yamamoto H, Isoda N, et al: Development of gastric carcinoma from intestinal metaplasia in Cdx2-trasgenic mice. Cancer Res 64: 7740-7747, 2004.

17. Li MK and Folpe AL: CDX-2, a new marker for adenocarcinoma of gastrointestinal origin. Adv Anat Pathol 11: 101-105, 2004.

18. Guo RJ, Suh ER and Lynch JP: The role of Cdx proteins in intestinal development and cancer. Cancer Biol Ther 3: 593-601, 2004.

19. Kato R, Murata K, Okamura S, Wada Y, Makino S, Nishigaki T, Owada Y, Murakami M, Okada K, Ebisui C, et al: Resection of ovarian metastasis of colon cancer to improve quality of life. Gan To Kagaku Ryoho 39: 2278-2279, 2012 (In Japanese).

20. McCormick CC, Giuntoli RL II, Gardner GJ, Schulick RD, Judson K, Ronnett BM, Vang R and Bristow RE: The role of cytoreductive surgery for colon cancer metastatic to the ovary. Gynecol Oncol 105: 791-795, 2007.

21. Jiang R, Tang J, Cheng X and Zang RY: Surgical treatment for patients with different origins of Krukenberg tumors: Outcomes and prognostic factors. Eur J Surg Oncol 35: 92-97, 2009.

22. Saltz LB, Clarke S, Díaz-Rubio E, Scheithauer W, Figer A, Wong R, Koski S, Lichinitser M, Yang TS, Rivera F, et al: Bevacizumab in combination with oxaliplatin-based chemotherapy as first-line therapy in metastatic colorectal cancer: A randomized phase III study. J Clin Oncol 26: 2013-2019, 2008.

23. Fuchs CS, Marshall J and Barrueco J: Randomized, controlled trial of irinotecan plus infusional, bolus, or oral fluoropyrimidines in first-line treatment of metastatic colorectal cancer: Updated results from the BICC-C study. J Clin Oncol 26: 689-690, 2008.

24. Douillard JY, Siena S, Cassidy J, Tabernero J, Burkes R, Barugel M, Humblet Y, Bodoky G, Cunningham D, Jassem J, et al: Randomized, phase III trial of panitumumab with infusional fluorouracil, leucovorin and oxaliplatin (FOLFOX4) versus FOLFOX4 alone as first-line treatment in patients with previously untreated metastatic colorectal cancer: The PRIME study. J Clin Oncol 28: 4697-4705, 2010.

25. Köhne CH, Hofheinz R, Mineur L, Letocha H, Greil R, Thaler J, Fernebro E, Gamelin E, Decosta L and Karthaus M: First-line panitumumab plus irinotecan/5-fluorouracil/leucovorin treatment in patients with metastatic colorectal cancer. J Cancer Res Clin Oncol 138: 65-72, 2012. 